\title{
EI Desarrollo De La Coordinación En Los Fundamentos Técnicos Del Fútbol En La Categoría 10-12 Años. Estudio De Caso: Test De Coordinación En La Escalera
}

Orlando David Mazón Moreno

Magister en Cultura Física y Entrenamiento Deportivo

Docente de la Escuela Superior Politécnica de Chimborazo, Ecuador

Jorge Giovanny Tocto Lobato

Magister en Cultura Física y Deportología

Docente de la Escuela Superior Politécnica de Chimborazo, Ecuador

Marlon Gabriel Llanga Colcha

Licenciado en Cultura Física y Entrenamiento Deportivo, Ecuador

Ramón Fernando Bayas Machado

Magister en Cultura Física y Deportología

Docente de la Universidad Nacional de Chimborazo, Ecuador

Juan Carlos Bayas Machado

Licenciado en Cultura Física y Entrenamiento Deportivo, Ecuador

doi: 10.19044/esj.2017.v13n23p206 URL:http://dx.doi.org/10.19044/esj.2017.v13n23p206

\begin{abstract}
The aim of this study was to determine the development of coordination and its impact on the technical fundamentals of football in the $10-12$ years of age category in "Unidad Educativa Liceo Policial Chimborazo" by a guide of exercises in order to improve coordination and soccer technical fundamentals for six weeks. For this research 25 players were evaluated. A pre and post-test were applied for coordination on the stairs. The results acquired in the implementation of the excise guide coordination and technical foundations of football were significant at the time of implementation of the post test. The exercise used in this guide and applied training sessions were coordinating the ball with the food. It has been concluded that coordination affects within the basic foundation of soccer and improve their lifestyle.
\end{abstract}

Keywords: Coordination, driving, exercises, grounds, training, players, guide, test 


\section{Resumen}

El objetivo de la presente investigación fue determinar el desarrollo de la coordinación y su incidencia en los fundamentos técnicos del fútbol en la categoría 10-12 años de la Unidad Educativa Liceo Policial Chimborazo mediante una guía de ejercicios para mejorar la coordinación y los fundamentos técnicos del futbol, durante seis semanas. Para esta investigación fueron evaluados 25 futbolistas, donde se aplicó un pre y post test de la coordinación en la escalera. Los resultados que se adquirieron en la aplicación de la guía de ejercicios de coordinación y fundamentos técnicos del futbol fueron muy significativos en el momento de la aplicación del post test. Los ejercicios utilizados en esta guía y aplicados en las sesiones de entrenamiento fueron de coordinación con el pie Se concluyó que la coordinación influye dentro de las fundamentaciones básicas del futbol y así mejorar su estado de vida.

Palabras-claves: Coordinación, conducción, ejercicios, escalera, entrenamiento, futbolistas, guía, test

\section{Introducción}

El fútbol, es lejos el deporte más popular en el mundo. No hay país, en el cual no se practique este deporte. De la misma manera, al fútbol, se le llama el deporte rey. "El fútbol es considerado como el elemento esencial a través de cual se puede interpretar y entender el avance y desarrollo de la sociedad ya que por medio de este deporte se logra la formación de seres integrales".

Según (Morales A. \& Guzmán M, 2000) definen como; "Un juego colectivo entre dos equipos de 11 jugadores cada uno, que requiere el dominio del propio cuerpo, cuya finalidad es hacer entrar un balón por una portería, basándose conforme a reglas determinadas, de las que la más característica es la prohibición de que sea tocado con las manos salvo por un jugador que guarda la portería, y este en una determinada zona”.

Para Hernández Moreno. J, (1993), el fútbol es un deporte de equipo de colaboración oposición, que se juega en un espacio semisalvaje y común, con participación simultánea. El desarrollo de la acción de juego depende de las acciones individuales y colectivas realizadas en una situación de colaboración con los compañeros y de oposición con los adversarios, de acuerdo con un pensamiento táctico individual que debe ser coordinado con el resto de los compañeros (págs. 181-191).

El presente trabajo describe la importancia del desarrollo de la coordinación en los fundamentos técnicos de fútbol ya que es uno de los deportes más populares a nivel mundial y por ende en nuestro país. El objetivo de este trabajo es investigar "El desarrollo de la Coordinación en los 
Fundamentos Técnicos de Fútbol en la categoría 10-12 años de la Unidad Educativa Liceo Policial Chimborazo, periodo febrero - agosto 2016".

Con esta investigación se pretende demostrar en la teoría y en la práctica la influencia y la importancia de las capacidades físicas básicas del fútbol pues permite desarrollar los aspectos técnico, táctico, físico, psicológico y social. El deporte es de suma importancia para nuestros hijos; en primer lugar, a nivel psicológico, el deporte les ayudará a enfrentarse a la competición, a plantearse diferentes metas, los hará más fuerte y les ayudará en algo tan importante como saber trabajar en equipo y comunicarse (Grisales J, 1994).

De acuerdo al autor Araujo (2002), manifiesta que "El fútbol es considerado como el elemento esencial a través de cual se puede interpretar y entender el avance y desarrollo de la sociedad ya que por medio de este deporte se logra la formación de seres integrales" además, los fundamentos técnicos del fútbol son el instrumento principal en la práctica deportiva, y le son dotados al jugador en el proceso de enseñanza aprendizaje; los fundamentos en un principio se realizarán en el propio terreno para ir agregando espacio en la cancha en movimiento.

Los principios tácticos o de juego son definidos como "ideas fundamentales básicas y esenciales del fútbol, que se han agrupado en un verdadero método de trabajo, que persigue dos finalidades específicas:

- $\quad$ Preparar al jugador para desenvolverse con éxito ante los numerosos problemas tácticos que a cada instante le presenta el fútbol durante la competencia.

- $\quad$ Formar en el jugador una mentalidad táctica o de juego que le permita tomar iniciativas o variar planes de juego, de acuerdo al acontecer de un partido, desarrollándose por medio de tres mecanismos (percepción decisión - ejecución) la inteligencia de juego.

Según Massafret (2010), manifiesta que: la coordinación es el conjunto de capacidades que favorecen la eficiencia y la adaptación del movimiento a las condiciones del entorno a través de la estrecha relación del sistema nervioso central y la musculatura esquelética, fomentando la individualidad y la creatividad en el gesto, favoreciendo el aprendizaje y facilitando el acercamiento entre la acción final real y la acción final deseada.

La coordinación va a influir de forma decisiva sobre la velocidad y la calidad de los procesos de aprendizajes de destrezas y técnicas específicas, que más tarde harán su aparición en el mundo escolar. Es por ello que la coordinación es una cualidad neuromuscular íntimamente ligada con el aprendizaje y que está determinada, sobre todo, por factores genéticos (Rivas y Sánchez, 2013). 
Rivera (2015), da a conocer tres funciones básicas de las capacidades coordinativas: condiciona la vida en general, condiciona el aprendizaje motor y condiciona el rendimiento deportivo, este último se define como el desarrollo de la coordinación en estas actividades y no es la rapidez ni cantidad de ejercicios realizados, sino la calidad del trabajo efectuado con el adulto mayor. Es mucho más importante el tanteo del adulto para resolver el problema que se le propone que los resultados obtenidos en el mismo.

Según Serrano (2013), a continuación se citan algunas de las posibles actividades para el desarrollo de la coordinación:

- Desplazamientos variados: (pata coja, cuadrupedia, reptar, trepar, etc.)

- Saltos de todo tipo: pies juntos, sobre un pie, alternativos, rítmicos, etc.

- Gestos naturales: tirar, transportar, empujar, levantar, etc.

Todas estas actividades tipo se pueden realizar utilizando diferentes direcciones, velocidades, ritmos, combinaciones, etc., o con una gran variedad de materiales complementarios. Este tipo de entrenamiento debe realizarse por 15 a 20 minutos, dos o tres veces a la semana. (Ejercicios I y II).

Actualmente el fútbol es un lenguaje universal de millones de personas en todo el mundo, incluidos niños, niñas y adolescentes, independientemente de su lugar de origen, idioma que hablen o religión a la que pertenezcan, en el entrenamiento del fútbol, cada día requiere de más importancia la pedagogía y los lineamientos metodológicos para orientar correctamente los contenidos de trabajo que se desarrollan en las diferentes etapas de formación, por lo tanto el fútbol como deporte colectivo complejo y competitivo constantemente presenta situaciones de juego cambiantes que exige al deportista percibir, analizar y decidir adecuadamente la solución más acertada para dichas ocasiones.

A cada uno de los deportes le corresponde un grado de importancia distinta ya que se intenta alcanzar el movimiento perfecto. En los juegos deportivos y también en el fútbol una técnica muy elaborada junto a una flexibilidad óptima le facilita al jugador resolver con éxito las situaciones de juego, es decir el proceso de resolución para la ejecución de la acción del movimiento óptimo, por parte del jugador.

En el Ecuador en los últimos años a través de organizaciones estatales principalmente por el MIES, Ministerio de Educación y el Ministerio de Deportes a través de estrategia se ha visto y analizado la necesidad de incluir a los niños y niñas a la práctica deportiva dentro de diferentes áreas, recreativas y deportivas realizando campañas, sociales, deportivas y educativas con el fin de rescatar a los niños y niñas de las calles y sobre todo permitir que tengan una educación digna y adecuada, orientando 
a los niños en la toma ágil y eficiente de decisiones para enfrentar los problemas que se presenten en la vida cotidiana.

\section{La escalera de coordinación}

La mayoría de los deportes que utilizan algún segmento corporal en el juego utilizan la escalera de coordinación, que puede adaptarse fácilmente con aros e incluso marcas pintadas en el suelo. La multitud de ejercicios de coordinación que pueden llevarse a cabo en la escalera hace que el entrenamiento sea tan dinámico como eficaz pudiéndolo combinarlo, además, con la integración de golpeos técnicos para una mejor disociación segmentaria.

\section{Evaluación de la coordinación}

No es fácil establecer test o pruebas útiles para medir y evaluar la coordinación al estar esta habilidad unida a otras capacidades. Aun así se pueden establecer dos apartados que nos ayuden a ver el nivel de esta capacidad

Test: nos referimos a examen psicomotor de Mazzo y de Vayer y a la observación psicomotriz de Da Fonseca.

Pruebas escolares: En este sentido existen diversas pruebas para evaluar la coordinación, pero en la práctica el docente utiliza el salto en profundidad, triple salto a pies juntos, etc.

\section{Test de la Escalera (Coordinación Óculo- Pédica)}

Este test se utiliza para evaluar la coordinación óculo pédica, además de la general o gestual. Consiste en correr por una escalera situada en el suelo formada por listones de madera o cuerda, los cuales están unidos por una cinta por ambos extremos.

Según los test realizados se ha tenido en cuenta el estudio llevado a cabo por (Martínez, 2003) sobre test de cualidades físicas y motrices. En esta investigación se actuó simultáneamente en tres frentes, cada uno de ellos estaba directamente relacionado con el estudio de las pruebas físicas. (EFFECTS 262, 2004-2005) en el tema la Evaluación de la Condición Física que se destaca en el siguiente cuadro.

Cuadro 1. Evaluación de la condición física

\begin{tabular}{|c|l|c|c|c|}
\hline GENERO & ALTO & NORMAL & BANO & DEFICIENTE \\
\hline MASCULINO & $<3^{\prime \prime}$ & $3^{\prime \prime} \mathrm{a} 4^{\prime}$ & $4^{\prime \prime} \mathrm{a} 5^{\prime}$ & $>5^{\prime}$ \\
\hline FEMENINO & $<^{\prime}$ & $4^{\prime \prime} \mathrm{a} 5^{\prime}$ & $5^{\prime \prime} \mathrm{a} 6^{\prime}$ & $>6^{\prime}$ \\
\hline
\end{tabular}

Fuente: (EFFECTS 262, 2004-2005) 
Estos son los resultados que tomamos como referencia, para poder realizar nuestro análisis, es importante decir que lo adaptamos a la edad del niño ya que los resultados que se obtuvieron en estos estudios fueron a deportistas profesionales.

\section{Propósito}

Evaluar la coordinación óculo pédica.

\section{Descripción del test}

El niño se coloca justo detrás de la 1a banda sin tocar la banda y a la señal sale a máxima velocidad hacia el extremo opuesto, debe realizar de ida y vuelta. Deberá realizarse un apoyo en cada espacio de separación entre bandas sin tocar dichas bandas. El cronómetro se detiene al pasar la última banda.

\section{Resultados}

Registrar el tiempo empleado en finalizar el test en segundos. Los resultados se comparan con los valores de referencia, que aparecen en la siguiente imagen, para determinar si la coordinación óculo - pédica es adecuada.

\section{Materiales}

- Escalera (ladder) de 20 bandas y 9 metros de largo, con una separación entre bandas de $40 \mathrm{~cm}$.

- Cronómetro.

\section{Puntaje}

Este test que se desarrolla en el niño dura un máximo de 15 segundos.

Tabla 1.

\begin{tabular}{cc} 
RESULTADO & FRECUENCIA \\
\hline Alto & $<10$ segundos \\
\hline Bajo & $\geq 15$ segundos \\
\hline Normal & $10-14$ segundos
\end{tabular}

Fuente: Categoría 10-12 años de la Unidad Educativa Liceo Policial Chimborazo

Elaborado por: Marlon Llanga

\section{Objetivo general}

Determinar la incidencia de la Coordinación en los Fundamentos Técnicos de Fútbol en la categoría 10-12 años de la Unidad Educativa Liceo Policial Chimborazo, periodo febrero - agosto 2016"

\section{Objetivos específicos}

- Analizar el nivel de coordinación en los niños de la categoría 10-12 años de la de la Unidad Educativa Liceo Policial Chimborazo. 
- $\quad$ Aplicar un pre y post test de los fundamentos técnicos de fútbol a los niños de la categoría 10-12 años de la de la Unidad Educativa Liceo Policial Chimborazo.

- Diseñar una guía de ejercicios para mejorar la coordinación y los fundamentos técnicos de fútbol de los niños de la categoría 10-12 años de la Unidad Educativa Liceo Policial Chimborazo.

\section{Metodología}

Es una investigación de campo y bibliográfica pues la información obtenida se la hizo directamente en el lugar de los hechos, es decir con los seleccionados de la Unidad Educativa "Liceo Policial Chimborazo" categorías 10-12 años, permitiéndonos que estemos seguros de las condiciones reales en que se han conseguido los datos.

Para este proyecto se consideró el tipo de estudio Transversal, porque está determinado según el período de tiempo en que se desarrolla, pues apunta a un momento y tiempo definido.

Para el procesamiento de la información usaremos los programas de Microsoft Word y Excel. Los resultados obtenidos se los presentara en gráficos y cuadros estadísticos con su respectivo análisis e interpretación.

\section{Población y muestra}

\begin{tabular}{ccc} 
Datos & $\begin{array}{c}\text { Tabla 1. Demostración de la población } \\
\text { Frecuencia }\end{array}$ & Porcentaje \\
\hline Niños & 25 & $100 \%$ \\
\hline TOTAL & 25 & $100 \%$
\end{tabular}

Fuente: Equipo de investigación

En esta investigación no se obtendrá muestra debido a que la población es de 25 niños, por tanto, trabajaremos con el 100\% de la población.

\section{Resultados del pre-test de la coordinación y los fundamentos técnicos del} fútbol

Test 1: test de la escalera (coordinación óculo- pédica)

Tabla 2. Coordinación (PRE - TEST)

\begin{tabular}{ccc} 
Nivel & Deportistas & Porcentajes \\
\hline Alto & 4 & $16 \%$ \\
\hline Bajo & 11 & $44 \%$ \\
\hline Normal & 10 & $40 \%$ \\
\hline TOTAL & 25 & 100
\end{tabular}

Fuente: Categoría 10-12 años de la Unidad Educativa Liceo Policial Chimborazo

Elaborado por: Equipo de investigación 


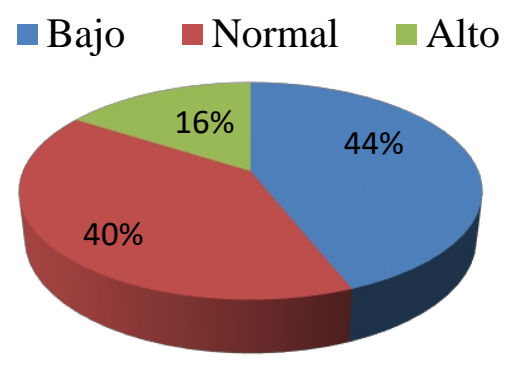

Gráfico 1. Categoría 10-12 años de la Unidad Educativa Liceo Policial Chimborazo

Elaborado por: Equipo de investigación

\section{Análisis - Interpretación}

Según el test realizado en el pre-test los niños deportistas en el test de la escalera sobre la coordinación, en el nivel Alto el niño deportista tiene un porcentaje del $16 \%$, en el nivel Bajo que cuentan con 11 niños deportistas esta con el $44 \%$, en similitud en el nivel Normal el porcentaje del pre-test tiene un $40 \%$, por lo tanto es importante que el niño deportista realice movimientos ordenados y dirigidos a la obtención de un gesto técnico.

\section{Análisis estadísticos Test de Escalera (Pre-Test)}

Tabla 3. Datos de los 25 jugadores

\begin{tabular}{ccc} 
Jugador No & Tiempo Pre-Test & Calificación \\
\hline 1 & $19 \mathrm{seg}$ & Bajo \\
\hline 2 & $11 \mathrm{seg}$ & Normal \\
\hline 3 & $8 \mathrm{seg}$ & Alto \\
\hline 4 & $13 \mathrm{seg}$ & Bormal \\
\hline 5 & $20 \mathrm{seg}$ & Normal \\
\hline 6 & $14 \mathrm{seg}$ & Bajo \\
\hline 7 & $19 \mathrm{seg}$ & Normal \\
\hline 8 & $12 \mathrm{seg}$ & Normal \\
\hline 9 & $14 \mathrm{seg}$ & Bajo \\
\hline 10 & $20 \mathrm{seg}$ & Alto \\
\hline 11 & $8 \mathrm{seg}$ & Alto \\
\hline 12 & $9 \mathrm{seg}$ & Normal \\
\hline 13 & $14 \mathrm{seg}$ & Bajo \\
\hline 14 & $19 \mathrm{seg}$ & Bajo \\
\hline 15 & $19 \mathrm{seg}$ & Bajo \\
\hline 16 & $16 \mathrm{seg}$ & Bajo \\
\hline 17 & $20 \mathrm{seg}$ & Normal \\
\hline 18 & $13 \mathrm{seg}$ & Bajo \\
\hline 19 & $20 \mathrm{seg}$ & Alto \\
\hline 20 & $9 \mathrm{seg}$ & Normal \\
\hline 21 & $13 \mathrm{seg}$ & Normal \\
\hline 22 & $14 \mathrm{seg}$ & Bajo \\
\hline 23 & $18 \mathrm{seg}$ & Normal \\
\hline 24 & $12 \mathrm{seg}$ & Bajo \\
\hline 25 & $17 \mathrm{seg}$ & Chimb
\end{tabular}

Fuente: Categoría 10-12 años de la Unidad Educativa Liceo Policial Chimborazo.

Elaborado por: Equipo de trabajo 
Guía de ejercicios sobre el desarrollo de la coordinación en los fundamentos técnicos del futbol

Ejercicios de coordinación con escalera

Ejercicio No 1

Objetivo: Desarrollar la coordinación mediante ejercicios en escalera a categoría 10-12 años de la Unidad Educativa Liceo Policial Chimborazo.

Materiales: Escalera, Silbato, Cronómetro.

Descripción: Skiping medio sobre escalera con mucha frecuencia con dos apoyos. Alternativo derecha e izquierda.

Dosificación: 3(5rep x 2`pausa)

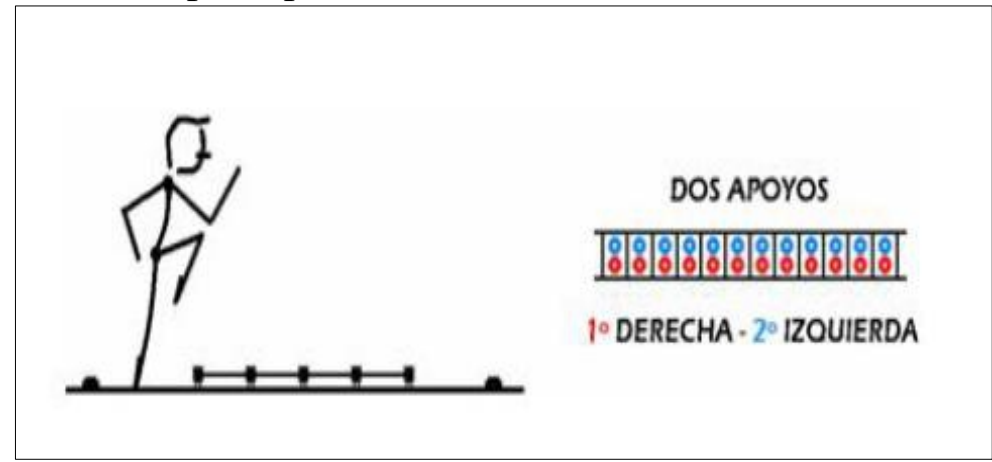

Gráfico 2. Descripción gráfica ejercicio 1

Fuente: http/futbolcarrasco.com/wp-

content/uploads/2014/07futbolcarrascoejerciciosfisicosjpg.

\section{Ejercicio No 2}

Objetivo: Desarrollar ejercicios de coordinación con un solo apoyo en escalera a la categoría 10-12 años de la Unidad Educativa Liceo Policial.

Materiales: Escalera, Silbato, Cronómetro.

Descripción: Skiping medio sobre escalera con mucha frecuencia con un solo apoyo alternativo derecha -izquierda

Dosificación: 3(5rep x 2`pausa)

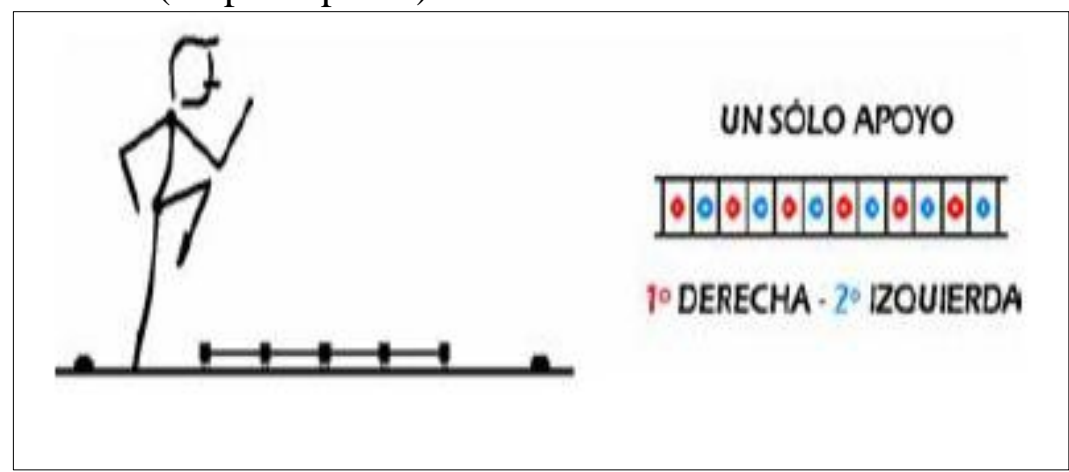

Gráfico 3. Descripción gráfica del ejercicio 2

Fuente: http/futbolcarrasco.com/wp-

content/uploads/2014/07futbolcarrascoejerciciosfisicosjpg 


\section{Ejercicio No 3}

Objetivo: Mejorar la coordinación de extremidades superiores e inferiores mediante ejercicios coordinados.

Materiales: Escalera, Silbato, Cronómetro.

Descripción: Desplazamiento lateral sobre escalera con mucha frecuencia con un apoyo dentro y otro fuera.

Dosificación: 3(5rep x 2`pausa)

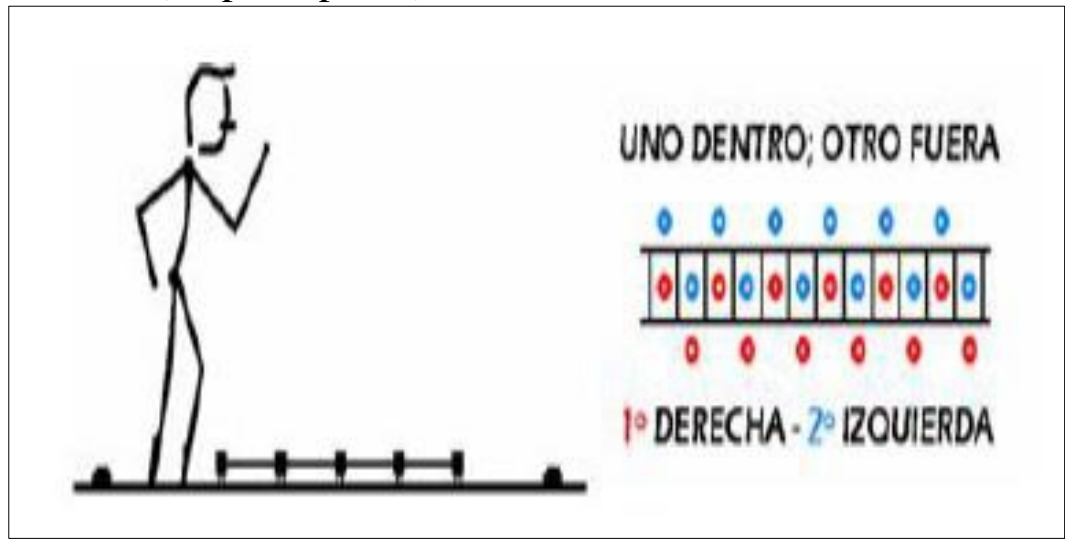

Gráfico 4. Descripción gráfica del ejercicio 3

Fuente: http/futbolcarrasco.com/wp-

content/uploads/2014/07futbolcarrascoejerciciosfisicosjpg

\section{Ejercicio No 4}

Objetivo: Desarrollar ejercicios de coordinación en escalera con la categoría 10-12 años de la Unidad Educativa Liceo Policial Chimborazo.

Materiales: Escalera, Silbato, Cronómetro.

Descripción: Skiping lateral sobre escalera con mucha frecuencia con doble apoyo delante y atrás.

Dosificación: 3(5rep x 2`pausa)

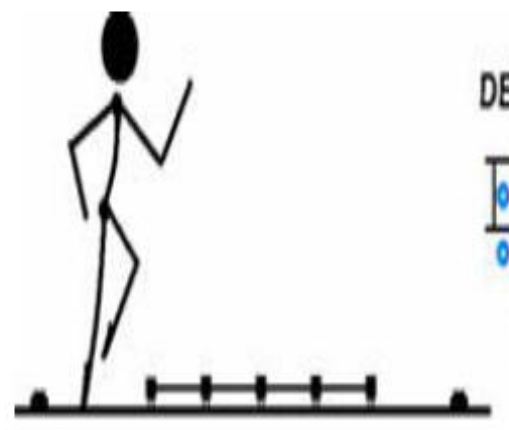

DELANTE-ATRAS DOBLEAPOYO

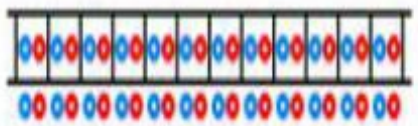

10 DERECHA. 2 I IOUIERDA

Gráfico 5. Descripción gráfica del ejercicio 4

Fuente: http/futbolcarrasco.com/wp-

content/uploads/2014/07futbolcarrascoejerciciosfisicosjpg 


\section{Ejercicio No 5}

Objetivo: Desarrollar ejercicios de coordinación en escalera con la categoría 10-12 años de la Unidad Educativa Liceo Policial Chimborazo.

Materiales: Escalera, Silbato, Cronómetro.

Descripción: Skiping lateral sobre escalera con mucha frecuencia con doble apoyo delante y atrás en diagonal.

Dosificación: 3(4rep x 2`pausa)

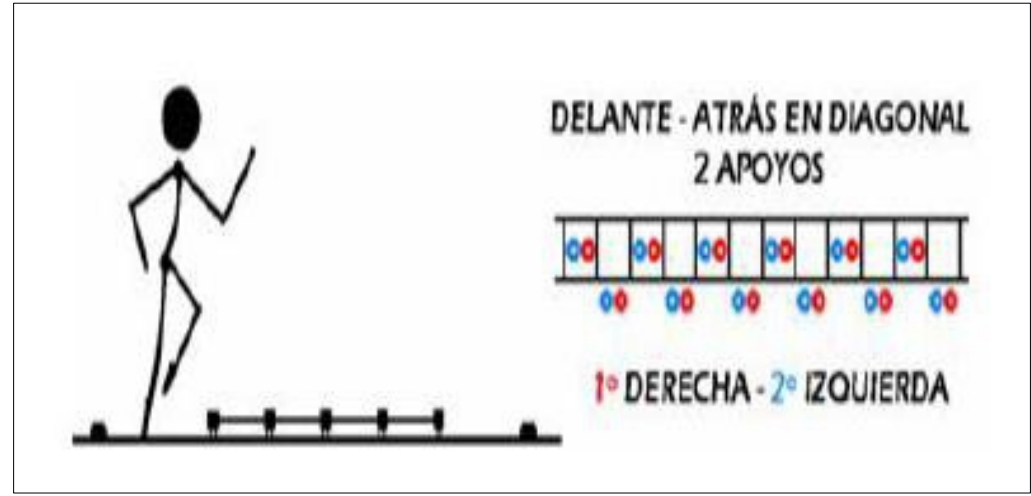

Gráfico 6. Descripción gráfica del ejercicio 5

Fuente: http/futbolcarrasco.com/wp-

content/uploads/2014/07futbolcarrascoejerciciosfisicosjpg

\section{Ejercicio No 6}

Objetivo: Desarrollar ejercicios de coordinación en escalera con la categoría 10-12 años de la Unidad Educativa Liceo Policial Chimborazo.

Materiales: Escalera, Silbato, Cronómetro.

Descripción: Desplazamiento lateral sobre escalera con mucha frecuencia y con apoyo fuera y doble dentro.

Dosificación: 3(5rep x 3`pausa)

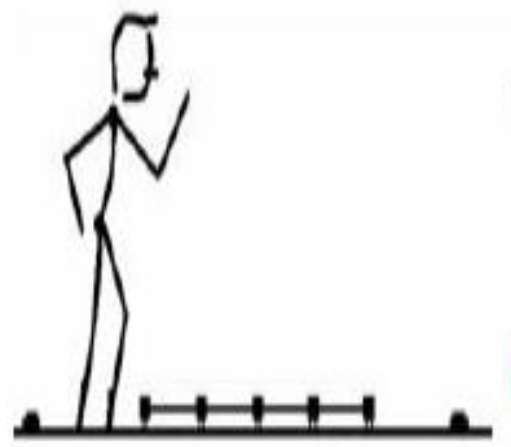

UNO FUERA DOBLE DENTRO

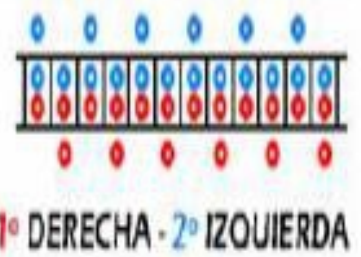

Gráfico 7. Descripción gráfica del ejercicio 6

Fuente: http/futbolcarrasco.com/wp-

content/uploads/2014/07futbolcarrascoejerciciosfisicosjpg 
Resultados del post-test de la coordinación y los fundamentos técnicos del fútbol

Test de Coordinación

\section{TEST No ${ }^{\circ}$ : Test de La Escalera (Coordinación Óculo- Pédica) Post-Test}

Tabla 4.

Coordinación (POST-TEST)

\begin{tabular}{ccc}
\hline Nivel & Deportista & $\%$ \\
\hline Alto & 8 & $32 \%$ \\
\hline Bajo & 6 & $24 \%$ \\
\hline Normal & 11 & $44 \%$ \\
\hline TOTAL & 25 & 100
\end{tabular}

Fuente: Categoría 10-12 años de la Unidad Educativa Liceo Policial Chimborazo

Elaborado por: Equipo de investigación

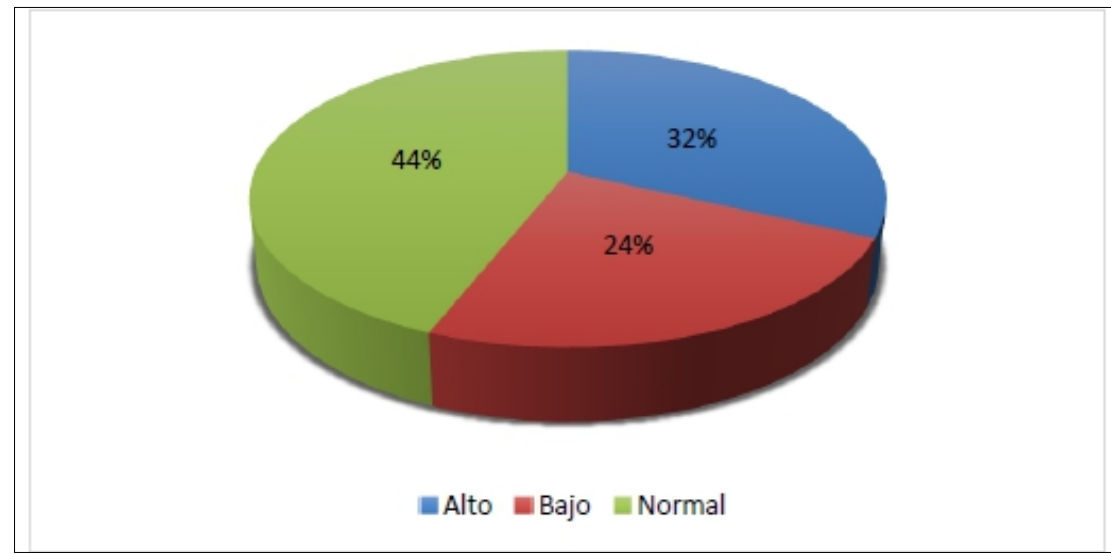

Gráfico 8. Descripción porcentual de los resultados del post test

Fuente: Categoría 10-12 años de la Unidad Educativa Liceo Policial Chimborazo

Análisis - Interpretación: Según los cuadros estadísticos y luego de los test anteriores en este pos-test podemos observar que los niños deportistas en el test de coordinación, en el nivel Alto el niño deportista tiene un porcentaje del 32\% duplicando su efectividad, en el nivel Bajo que cuentan con 6 niños deportistas esta con el $24 \%$, y en el nivel Normal el porcentaje en el pos-test tiene un $44 \%$, esto quiere decir que e se está trabajando en visón de mejorar las capacidades técnicas y movimientos dirigidos a la obtención de una calidad técnica 


\section{Análisis estadísticos Test de Escalera (Post-Test)}

Tabla 5.

Jugador No

Tiempo Post-test

Calificación

\begin{tabular}{|c|c|c|}
\hline 1 & $13 \mathrm{seg}$ & Normal \\
\hline 2 & $9 \mathrm{seg}$ & Alto \\
\hline 3 & $7 \mathrm{seg}$ & Alto \\
\hline 4 & $10 \mathrm{seg}$ & Normal \\
\hline 5 & $14 \mathrm{seg}$ & Normal \\
\hline 6 & $11 \mathrm{seg}$ & Normal \\
\hline 7 & $13 \mathrm{seg}$ & Normal \\
\hline 8 & $10 \mathrm{seg}$ & Normal \\
\hline 9 & $11 \mathrm{seg}$ & Normal \\
\hline 10 & $16 \mathrm{seg}$ & Bajo \\
\hline 11 & $6 \mathrm{seg}$ & Alto \\
\hline 12 & $8 \mathrm{seg}$ & Alto \\
\hline 13 & $9 \mathrm{seg}$ & Alto \\
\hline 14 & $15 \mathrm{seg}$ & Bajo \\
\hline 15 & $16 \mathrm{seg}$ & Bajo \\
\hline 16 & $9 \mathrm{seg}$ & Alto \\
\hline 17 & $16 \mathrm{seg}$ & Bajo \\
\hline 18 & $10 \mathrm{seg}$ & Normal \\
\hline 19 & $17 \mathrm{seg}$ & Bajo \\
\hline 20 & $7 \mathrm{seg}$ & Alto \\
\hline 21 & $11 \mathrm{seg}$ & Normal \\
\hline 22 & $12 \mathrm{seg}$ & Normal \\
\hline 23 & $15 \mathrm{seg}$ & Bajo \\
\hline 24 & $9 \mathrm{seg}$ & Alto \\
\hline 25 & $14 \mathrm{seg}$ & Normal \\
\hline
\end{tabular}

Fuente: Categoría 10-12 años de la Unidad Educativa Liceo Policial Chimborazo

Elaborado por: Equipo de trabajo

Resultados del pre-test y post-test de la coordinación y los fundamentos técnicos del fútbol

Test de coordinación

TEST Nº1: Test de la Escalera (Coordinación Óculo- Pédica)

Tabla 6.

COORDINACIÓN (ANTES)

\begin{tabular}{cccccc}
\hline Nivel & Deportista & $\%$ & Nivel & Deportista & $\%$ \\
\hline Alto & 4 & $16 \%$ & Alto & 8 & $32 \%$ \\
\hline Bajo & 11 & $44 \%$ & Bajo & 6 & $24 \%$ \\
\hline Normal & 10 & $40 \%$ & Normal & 11 & $44 \%$ \\
\hline TOTAL & 25 & 100 & TOTAL & 25 & 100
\end{tabular}

Fuente: Categoría 10-12 años de la Unidad Educativa Liceo Policial Chimborazo

Elaborado por: Equipo de investigación 


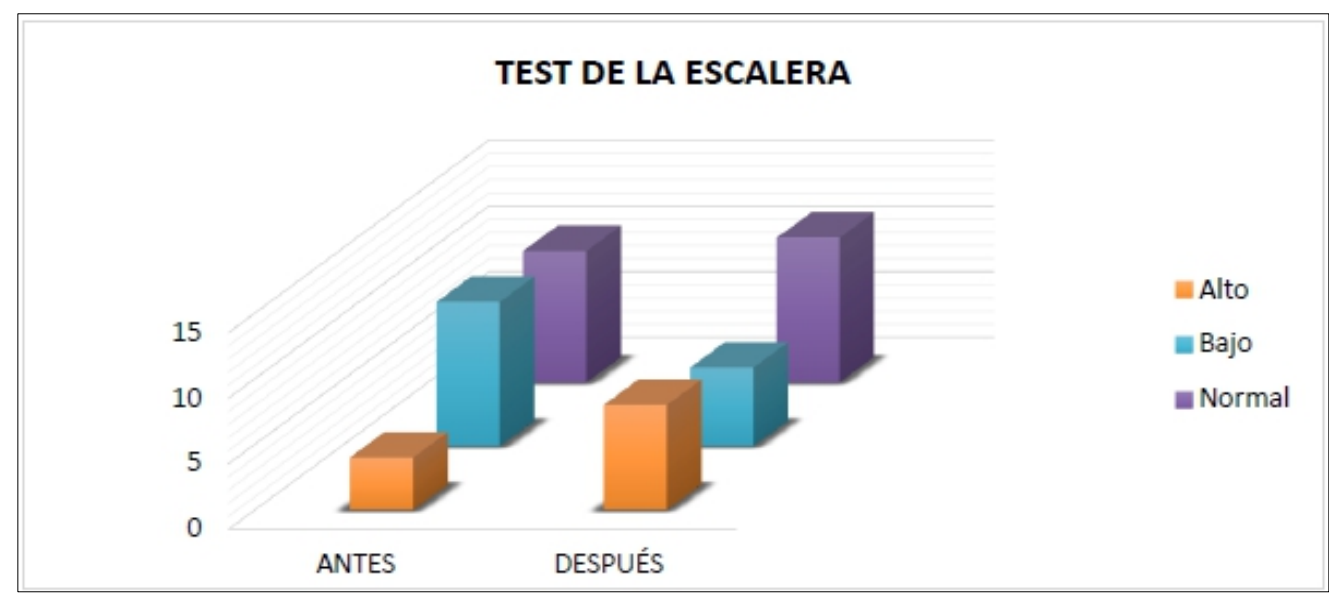

Gráfico 9. Descripción porcentual de resultados del pre y post test de escalera Fuente: Categoría 10-12 años de la Unidad Educativa Liceo Policial Chimborazo

\section{Análisis - Interpretación}

Se puede observar que en los Test realizados a los niños deportistas sobre la prueba de coordinación, en el parámetro Alto el porcentaje del postest ha mejorado teniendo un incremento al 32\%, de igual manera con el parámetro Normal el porcentaje del postest mejoro teniendo un $44 \%$, esto quiere decir que luego de un proceso de trabajo y entrenamiento a través de los ejercicios de coordinación van mejorando ya que los niños futbolistas se ejercitaron en el trabajo coordinativo en forma planificada.

\section{Análisis estadísticos Test de Escalera (Pre-Post Test)}

Tabla 7.

\begin{tabular}{ccccc}
$\begin{array}{c}\text { Jugador } \\
\text { No }\end{array}$ & $\begin{array}{c}\text { Tiempo } \\
\text { Pre-Test }\end{array}$ & Calificación & $\begin{array}{c}\text { Tiempo } \\
\text { Post-test }\end{array}$ & Calificación \\
\hline 1 & $19 \mathrm{seg}$ & Bajo & $13 \mathrm{seg}$ & Normal \\
\hline 2 & $11 \mathrm{seg}$ & Normal & $9 \mathrm{seg}$ & Alto \\
\hline 3 & $8 \mathrm{seg}$ & Alto & $7 \mathrm{seg}$ & Alto \\
\hline 4 & $13 \mathrm{seg}$ & Normal & $10 \mathrm{seg}$ & Normal \\
\hline 5 & $20 \mathrm{seg}$ & Bajo & $14 \mathrm{seg}$ & Normal \\
\hline 6 & $14 \mathrm{seg}$ & Normal & $11 \mathrm{seg}$ & Normal \\
\hline 7 & $19 \mathrm{seg}$ & Bajo & $13 \mathrm{seg}$ & Normal \\
\hline 8 & $12 \mathrm{seg}$ & Normal & $10 \mathrm{seg}$ & Normal \\
\hline 9 & $14 \mathrm{seg}$ & Normal & $11 \mathrm{seg}$ & Normal \\
\hline 10 & $20 \mathrm{seg}$ & Bajo & $16 \mathrm{seg}$ & Bajo \\
\hline 11 & $8 \mathrm{seg}$ & Alto & $6 \mathrm{seg}$ & Alto \\
\hline 12 & $9 \mathrm{seg}$ & Alto & $8 \mathrm{seg}$ & Alto \\
\hline 13 & $14 \mathrm{seg}$ & Normal & $9 \mathrm{seg}$ & Alto \\
\hline 14 & $19 \mathrm{seg}$ & Bajo & $15 \mathrm{seg}$ & Bajo \\
\hline 15 & $19 \mathrm{seg}$ & Bajo & $16 \mathrm{seg}$ & Bajo \\
\hline 16 & $16 \mathrm{seg}$ & Bajo & $9 \mathrm{seg}$ & Alto \\
\hline 17 & $20 \mathrm{seg}$ & Bajo & $16 \mathrm{seg}$ & Bajo \\
\hline 18 & $13 \mathrm{seg}$ & Normal & $10 \mathrm{seg}$ & Normal
\end{tabular}




\begin{tabular}{ccccc}
19 & $20 \mathrm{seg}$ & Bajo & $17 \mathrm{seg}$ & Bajo \\
\hline 20 & $9 \mathrm{seg}$ & Alto & $7 \mathrm{seg}$ & Alto \\
\hline 21 & $13 \mathrm{seg}$ & Normal & $11 \mathrm{seg}$ & Normal \\
\hline 22 & $14 \mathrm{seg}$ & Normal & $12 \mathrm{seg}$ & Normal \\
\hline 23 & $18 \mathrm{seg}$ & Bajo & $15 \mathrm{seg}$ & Bajo \\
\hline 24 & $12 \mathrm{seg}$ & Normal & $9 \mathrm{seg}$ & Alto \\
\hline 25 & $17 \mathrm{seg}$ & Bajo & $14 \mathrm{seg}$ & Normal
\end{tabular}

Fuente: Categoría 10-12 años de la Unidad Educativa Liceo Policial Chimborazo.

Elaborado por: Equipo de investigación

\section{Conclusion}

Se analizó el test coordinativo a la categoría de 10-12 años de edad de la Unidad Educativa Liceo Policial Chimborazo para medir el impacto de la coordinación a través de los fundamentos básicos del fútbol, el $32 \%$ de los niños han mejorado y desarrollado las capacidades coordinativas.

Luego de haber aplicado el pre-test y el post- test de los fundamentos técnicos del futbol a la categoría 10-12 años de la Unidad Educativa Liceo Policial Chimborazo, el niño va adquiriendo y mejorando el gesto deportivo y cualidades coordinativas, mediante técnicas y estrategias que ayuden a su rendimiento óptimo mediante trabajos progresivos e intensos ya que diversos niños deportistas tenían una descoordinación parcial en los movimientos técnicos específicamente al momento de trabajar con el balón.

Como podemos ver luego de la aplicación del test sobre los Fundamentos Técnicos de Fútbol de la categoría de fútbol de 10-12 años de edad de la Unidad Educativa Liceo Policial Chimborazo, han mejorado el nivel de coordinación dentro de fundamentos básicos del fútbol en un $44 \%$, por lo tanto esta investigación nos permite tener una clara visión de las actividades que se quiere realizar para mejorar el proceso de entrenamiento y utilizar metodologías y técnicas adecuadas en el desarrollo del futbol base.

\section{References:}

1. Araujo S. (2002). Flexiteste: Proposição de cinco índices de variabilidade da mobilidade articular. Revista Brasileira de Medicina do Esporte, 8(1):13-19.

2. Grisales J. (1994). Como mejorar la técnica individual del futbolista.". Medellín-Colombia.: Editorial I.G.M.

3. Hernández Moreno. J. (1993). Una metodología de la observación de juego en el fútbol. Cuantificación del tiempo de pausa y de participación. Ciencia y técnica del fútbol. Madrid: Gymnos.

4. Martínez E. (2003). La Flexibilidad: pruebas aplicables en educación secundaria - grado de utilización del profesorado. Consultado el 27/05/2017. Disponible en: Revista Digital, Educación Física y Deportes - http//:www.efdeportes.com 
5. Massafret M. (2010). Procesos coordinativos, optimización de la técnica, Apuntes del master profesional en alto rendimiento deportivo de deportes de equipo. Barcelona.

6. Morales A. \& Guzmán M. (2000). Diccionario Temático de los Deportes. . Málaga: Arguval.

7. Rivas M., Sánchez E. (2013). Fútbol. Entrenamiento actual de la condición física del futbolista; La coordinación en el futbolista. Consultado el 26/05/2017. Disponible en: http://www.redalyc.org/pdf/2370/237029450003.pdf

8. Rivera D. (2015). Diseño de pruebas motrices coordinativas. Hacia la autoevaluación en educación física. Consultado el 27/05/2017. Disponible en: http://www.tdx.cat/bitstream/handle/10803/312825/Tdrn1de1.pdf;jse ssionid=42CB7DE5239115619211C15F8F970950? sequence $=5$

9. Serrano E. (2013). La coordinación motriz y su incidencia en el desarrollo de la motricidad gruesa de los estudiantes del décimo año de educación básica del Instituto Tecnológico Superior "María Natalia Vaca" de la ciudad de Ambato - Ecuador. Consultado el 27/05/2017. Disponible en: http://repo.uta.edu.ec/bitstream/123456789/8799/1/FCHE-CFP323.pdf 